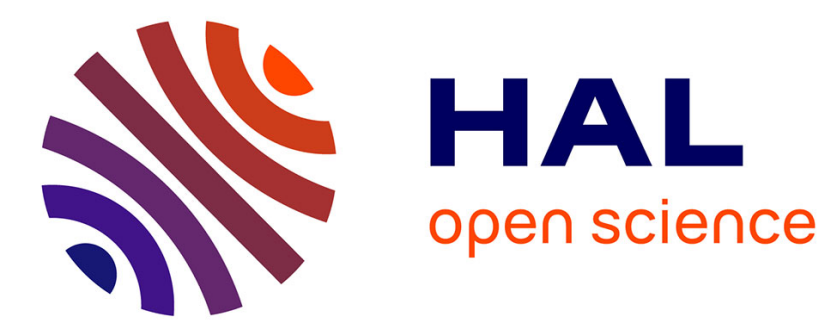

\title{
Wavelet compression of digital holograms: Towards a view-dependent framework
}

Kartik Viswanathan, Patrick Gioia, Luce Morin

\section{To cite this version:}

Kartik Viswanathan, Patrick Gioia, Luce Morin. Wavelet compression of digital holograms: Towards a view-dependent framework. SPIE Conference on Applications of Digital Image Processing XXXVI, Aug 2013, San Diego, United States. 10.1117/12.2027199 . hal-01229595

\section{HAL Id: hal-01229595 \\ https://hal.science/hal-01229595}

Submitted on 17 Nov 2015

HAL is a multi-disciplinary open access archive for the deposit and dissemination of scientific research documents, whether they are published or not. The documents may come from teaching and research institutions in France or abroad, or from public or private research centers.
L'archive ouverte pluridisciplinaire HAL, est destinée au dépôt et à la diffusion de documents scientifiques de niveau recherche, publiés ou non, émanant des établissements d'enseignement et de recherche français ou étrangers, des laboratoires publics ou privés. 


\title{
Wavelet compression of Digital Holograms: Towards a view-dependent framework
}

\author{
Kartik Viswanathan $^{a}$, Patrick Gioia ${ }^{a}$ and Luce Morin $^{b}$ \\ ${ }^{a}$ Orange Labs, Rennes, France; \\ ${ }^{b}$ INSA, Rennes, France;
}

\begin{abstract}
An analysis and discussion on the relevance of various wavelet schemes for hologram compression and reconstruction when the rendering configuration makes it possible to exploit selective refinements to perform a viewpointbased degraded reconstruction. It is observed that Gabor wavelet bases have better time-frequency localization as compared to Fresnelet bases and hence are well suited for view-dependent compression techniques for hologram reconstruction.
\end{abstract}

Keywords: Gabor wavelets, Fresnelets, Digital Holography, View-dependent Compression

\section{INTRODUCTION}

Holography is one principle candidate to the emergence of true 3D displays, i.e. providing full parallax. With the advance of computer power, screen resolutions and network bandwidths, the use of new digital methods have made possible the rise of animated full color holographic videos, as opposed to the static monochromatic classical optical process.

The hardware and network improvements haven't tackled the technical challenges, among which the compression issues hold an important place. The reason for this is that the amount of data for encoding holographic content (and more generally massively multi-view data) is considerable since targeted parallax quality should allow solving the convergence / accommodation mismatch, which implies the equivalent of thousands of distinct views for a single video frame.

There have been several attempts to compress holographic videos. The straightforward approaches such as applying block based video coding methods ${ }^{1}$ or pixel-based wavelets ${ }^{2}$ haven't come up with satisfactory results since they clearly don't take into account the special frequency characteristics of holograms; indeed, holograms are diffraction patterns that can have local frequencies of the order of magnitude of the coherent light wavelength used for their reconstruction.

More advanced methods were proposed in order to extract these frequency information from the raw local encoding; stereograms ${ }^{3}$ allow to represent holograms as a sum of piecewise constant frequency patterns. While impressive compression rates are obtained with such technique, the phase information is generally too degraded to form clearly focused images on the retina, requiring the use of phase smoothing post-process. ${ }^{4}$

With regards to phase continuity, wavelets could be seen as an improvement of the stereogram paradigm since it also compactly describes the frequency components of the data, but also provides a functional framework suited to smoothness and efficient encoding algorithms. Nonetheless, the special nature of the encoded data requires designing dedicated wavelets. These considerations have lead to Fresnelets, ${ }^{5}$ wavelets obtained by Fresnel transform of B-Spline wavelets. As discussed later in the article, Fresnelets do not show good localization in frequency which may be crucial for coefficient selection.

In ${ }^{6}{ }^{6}$ Gabor wavelets were proposed and their ability to remove unwanted orders of diffraction under certain assumptions.

Further author information:

E-mail: Kartik.viswanathan@orange.com, Telephone: +33 (0)299124994

E-mail: Patrick.gioia@orange.com, Telephone: +33 (0)299124198

E-mail: Luce.Morin@insa-rennes.fr, Telephone: +33 (0)223238757 
In this article, we stress the fact that a good wavelet scheme for holography should allow to perform a coefficient pruning corresponding to a viewpoint selection; indeed, one important source of scalability with multi-view data is the scalability in viewpoint. This scalability has already been implemented in ${ }^{7}$ independently of compression considerations, and do not only allow to reduce the amount of data to be transmitted, but also to simplify the displayed pattern, which may be useful until display devices have sufficient resolution to render complete holograms. This pruning also simplifies the computational complexity of the hologram synthesis.

The goal of this paper is to analyze the limitations of Fresnelets regarding both compression and adaptive reconstruction and show why Gabor wavelets may appear to be the ideal candidate for adaptive reconstruction, and to suggest improvements towards the use of Gabor-like wavelets for efficient viewpoint-based scalability. Indeed, this kind of scalability has gathered little interest so far but may be determinant for effective holography deployment.

The rest of the paper is organized as follows. Section 2 talks about the wavelets used in hologram compression so far, and their shortcomings for view based compression systems. Section 3 stresses the importance of localization for view-dependent coding. In Sections 4 and 5 we discuss the suitability of Fresnelets and Gabor wavelets regarding this type of coding. Section 6 provides results of reconstruction using Fresnelets and Gabor wavelets and Section 7 is the conclusion.

\section{WAVELET BASED TRANSFORMS SO FAR}

Holograms are quite untypical data to encode, and hence the tools classically designed for data compression may not be straight forwardly applicable. The reason for this is that information that is localized in the 3D encoded scene is spread out in the entire hologram, under the form of very rapidly oscillating patterns. Hence wavelets, which take profit of local regularities, are not really suited to hologram coding in their classical form.

The basic criteria that a wavelet should fulfill are that it should be able to approximate the hologram reasonably well and capture the frequency information at each pixel location. Holograms are not smooth functions that can be approximated easily. Also, to capture the large frequency variations in a hologram, it is natural to expect a wavelet which is extremely well localized in time and frequency. This criterion is analogous to the quality of a holographic display based on the metric of Space-Bandwidth product $(S B P)$. More information on the Space-Bandwidth product can be obtained here.$^{8}$

So far the wavelet transforms for holograms are divided into two broad categories. The first category is the set of wavelets that fulfill the admissibility criteria (mathematically) and are in line with the principles of wavelet multi resolution analysis. The important thing about these wavelet is the time (space) - frequency analysis that can be possible. All generic wavelets like the Haar wavelet, Meyer wavelet, Coifflet, Daub-4 etc. fall under this category.

Although direct application of filter banks used for image compression have been applied to holograms ${ }^{2}$ in order to be efficient, wavelets have to be adapted, often by relaxing some requirements. This is necessary when designing wavelet-like functions by observing the close relation between the optical diffraction phenomenon and wave propagation to the scaling of wavelets, i.e the scale parameter $s$ denotes the distance (depth) of the propagation. This was first proposed by Onural in.$^{9}$ Though this theory is interesting in the sense that if we are ready to give up the multi-resolution analysis framework and focus on the space - depth analysis rather than time - frequency analysis, there are certain mathematical questions that remain unanswered.$^{10}$ Its has been shown by Onural in ${ }^{11}$ that the chirp function (chirplet), performs a transform which is reversible, but yet it is not a mathematically admissible wavelet.

In the same spirit, Fresnelets ${ }^{5}$ try to compensate the effect of wavefront propagation by bringing the Fresnel transform of the original signal into the wavelet analysis. This implies adapting some conditions on multiresolution analysis definition, but above all releasing properties of space and frequency localization.

On the other hand, if one wants to keep good space and frequency localization, this may be at the expense of computational properties. This is the case for Gabor wavelets, which in particular do not form a partition of unity. The acceptability of the loose conditions of Fresnelets and Gabor wavelets will be discussed later in the article. 


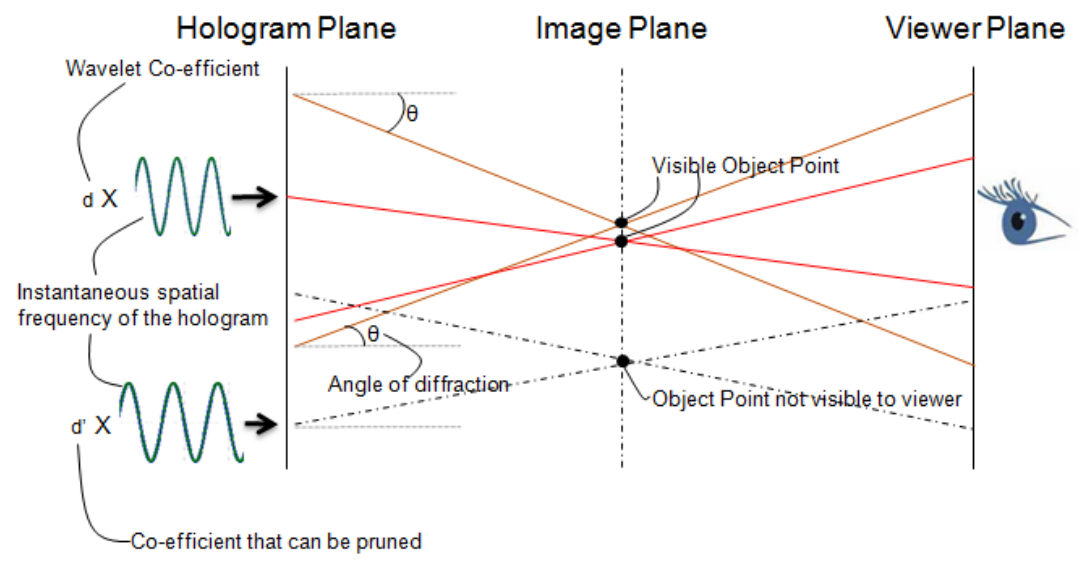

Figure 1. View-dependent compression of holograms

${ }^{2}$ provides an optimistic view of the wavelet based compression on holograms using several admissible wavelets. Compression using quantization and thresholding techniques of the wavelet coefficients is also shown. The results are formulated for simple holograms (usually single objects in scene) which do not hold a lot of spatial and frequency information. In actuality, standard wavelets which very capable of approximating piecewise smooth functions, tend to fail to capture the features of a hologram and hence provide poor results.

\section{VIEW-BASED COMPRESSION USING WAVELETS}

Although wavelets are a powerful tool for data compression, their special capability of local analysis of the signals make them especially suited to partial and adaptive reconstruction. This feature is classically used to degrade some data in space (or time) and frequency according to the final user requirements or rendering parameters. When it comes to holography, wavelets can efficiently help exploit a particular type of adaptivity, namely the adaptivity in point of view.

Holograms can be seen as a superimposition of patterns associated to specific directions of light emission. This property is widely exploited by stereograms, which isolate the various directions of diffraction. A partial reconstruction of a hologram can be made according to these pattern, as in , ${ }^{7}$ where they are denoted as $s u b$ holograms.

Such degradation is all the more important for digital holography so that it can be exploited not only at the stage of transmission, but also on the display side, with resolutions that would not allow to handle a full hologram. We thus see that in special cases, when only a restricted number of users are allowed to view the hologram, a good compression scheme may not be the one that provides the best absolute compression ratio, but the one that allows efficiently degrading the signal so as to transmit only the data relevant for identified observers. Figure 1 gives an example of such a compression scheme.

With regards to wavelets, such adaptation would mean pruning across the wavelet coefficients. Considering the well-known grating equation, the importance of good localization in space and frequency becomes obvious:

$$
\sin \theta=\frac{m \lambda}{\Lambda},
$$

where $\theta$ is the angle of diffraction, $\lambda$ the wavelength, $\Lambda$ the period of the grating and $m$ is the order of diffraction. 
We see that a coefficient will become relevant for a given propagation angle if it is related to the proper frequency according to this relation, i.e. the spectrum of these wavelets have to be concentrated around this value. On the other hand, wavelets have also to have good localization in space, since this directional diffraction is considered locally.

In the next sections we will discuss which wavelet bases provide this "good" localization which is best suited for this kind of compression technique.

\section{FRESNELETS}

Digital holography being a lensless process, will tend to spread out the sharp features like edges over the entire hologram plane. This spreading out of information is exploited in the formation of the Fresnelet bases. Fresnelets are formed by taking the Fresnel transform of appropriate B-Splines to simulate the propagation phenomenon (spreading out) in the hologram reconstruction process and use it as a wavelet bases.

If we were to use the wavelet coefficients that are generated after a Fresnel transform, the frequency information captured by them would be indistinguishable. Hence localization requirement of such wavelets is not very strong. It will then be useless for view-dependent hologram compression.

From the uncertainty relation of for Fresnel transforms ${ }^{5}$, the bound on the time-bandwidth product given as the product of the square of the variances for complex functions

$$
\sigma_{g}^{2} \sigma_{\tilde{g}}^{2} \leq \frac{\tau^{4}}{16 \pi^{2}}
$$

where $\sigma_{g}^{2}$ is the variance of the signal $g(x)$ and $\sigma_{\tilde{g}}^{2}$ is the variance of the fresnel transformed signal $\tilde{g}(x)$. In order to make this inequality an equality we need to have

$$
g(x)=a e^{i \omega_{o} x} e^{-b\left(x-x_{0}\right)} e^{-i \pi\left(x / \tau^{2}\right)}
$$

where $\tau=\sqrt{\lambda d}$ and $d$ is the distance of propagation.

Taking the Fresnel transform of this family of functions, we obtain the product of a chirp function of the form $e^{\alpha x^{2}}$ by a Gaussian function, which may be interpreted as its envelop. As shown in ${ }^{5}$ as parameter $\tau$ (which is directly proportional to the distance of propagation) increases, the spatial spreading of this envelop increases further. Since the chirp function frequency varies monotonically as we move away from the origin, more relevant frequency will then be taken into account. Since the function in equation (3) is the best possible case with respect to uncertainty relation, we see that this frequency spreading will occur for any arbitrary function.

The spatial spreading of the wavelet basis (due to Fresnel transform of the B-Splines) is another feature which is not well suited for view-based hologram compression techniques. It is imperative that there be a good localization in the spatial domain to capture the local features of the hologram. In ${ }^{5}$ it is shown that as the scale becomes finer, the Fresnelet bases tend to spread out (instead of becoming smaller) in the spatial domain. This eventually will lead to smaller number of bases functions on the hologram. Later extraction of the features of the hologram based on these fewer bases will result in poorer reconstruction for view-based compression systems, according to the requirements mentioned in Section 3. Figure 2 shows the time (spatial) domain spreading of the function for the finest scale.

Although theoretically proved that Fresnelets cannot have good localization in time and frequency, one may argue that in practice, they can be chosen close enough to functions that do have this property. This is countered by the following arguments:

It has been shown in, ${ }^{12}$ that B-Splines asymptotically converge to Gabor functions as the degree tends to $\infty$. Gabor functions are Gaussian functions which are modulated with a sinusoidal carrier. That means B-splines tend to converge to the form $e^{i 2 \pi m b x} g(x-n a)$ as the order of the B-spline goes to $\infty$.

If we make the assumption that the limit Gabor basis is a Riesz basis, then we can invoke the Bailen-Low ${ }^{13}$ theorem and deduce that this limit basis is not well-localized in time and frequency; the approximating series of B-Spline functions will then not behave better, not matter how close they approach the limit: 

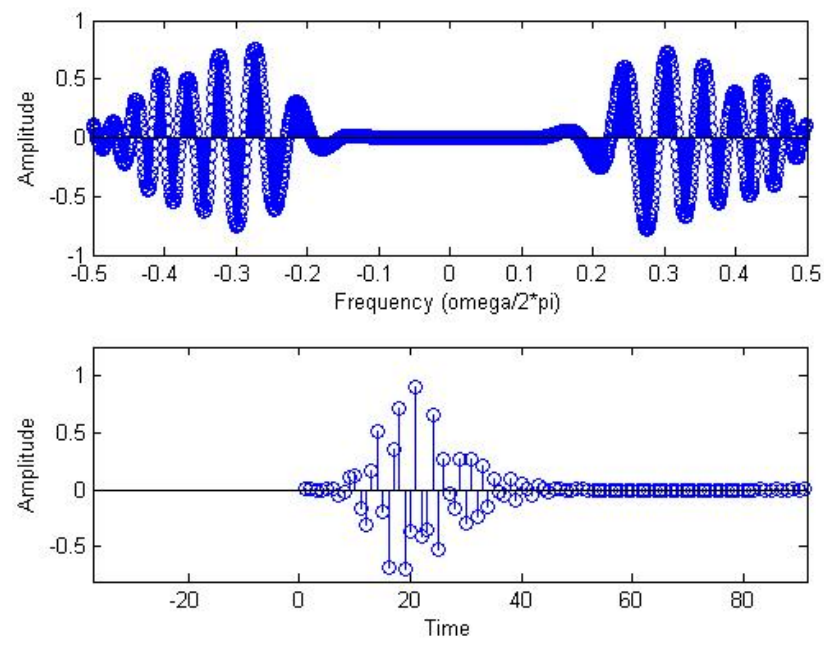

Figure 2. Fresnelet Basis showing the Frequency (top) and Time domain representations for the real part for the finest scale. Notice the spreading of the base in the time (spatial) domain

$$
\left(\int_{\mathbb{R}}|x g(x)|^{2} d x\right)\left(\int_{\mathbb{R}}|\gamma \hat{g}(\gamma)|^{2} d \gamma\right)=\infty
$$

Fresnelets which are Fresnel transforms of these B-Splines, will in fact spread the frequency further, and hence it can be said that Fresnelets cannot fulfill Balian-Low theorem.

$$
\int_{\mathbb{R}}|\gamma \hat{\tilde{g}}(\gamma)|^{2} d \gamma=\infty
$$

Figure 2 shows the spreading out of the frequency information in a Fresnelet with degree 3 . Such a large spread in the spatial and frequency domains of the Fresnelets is not well suited for application in view-dependent hologram compression.

Generally, holograms have the inherent problem of generating unwanted orders of diffraction, which tend to overlap with the required output. There are several solutions suggested in the literature of hologram generation. One of the methods is the generation of holograms using the phase-shift interferometry (PSI) in which an inline hologram is generated with natural suppression of the unwanted orders. The disadvantage being that the same hologram needs to be produced at atleast 3 different phases. Off-axis holography allows for separation of the orders in the image plane. But pixelated hologram storage in SLMs bring about a hard limitation to the maximum separation that can be obtained without overlapping with aliases.

One of the major problems that needs to be overcome in digital hologram reconstruction is the removal of unwanted orders. The intensity $I(x, y)$ of a hologram can be written as:

$$
I(x, y)=R \cdot R^{*}+O \cdot O^{*}+O \cdot R^{*}+O^{*} \cdot R
$$

where $R$ represents the reconstruction wave and $O$ represents the object wave.

The first and second term form the zero-order term. The third and fourth terms are the virtual and real image terms respectively. We are in general interested in the isolation of the virtual image, and the subsequent suppression of the zero-order and real image terms. To bring a separation between the three orders, usually the hologram is generated in off-axis configurations, after which the unwanted orders can be suppressed optically by using stops. Generic methods also include performing suppression in the frequency domain by bandpass filtering as the spatial frequencies of the orders are well separated from each other. 
Finally, among other properties, we can note that Fresnelets can bring about the suppression of the unwanted orders. In ${ }^{5}$ it is shown that the systematic modulation of the hologram, using the experiment parameters $k_{x}$ and $k_{y}$ which are the frequencies around which the real and virtual images are localized in the frequency domain, causes a shift of these images, such that the virtual image is localized at $(0,0)$ and the unwanted orders at $\left(k_{x}, k_{y}\right)$ and $\left(2 k_{x}, 2 k_{y}\right)$. The lower frequency information is located in the coarser sub-bands. Hence the coarser resolutions of the fresnelet decomposition tend to suppress the zero order and real image.

For further encoding of wavelet coefficients, ${ }^{14}$ propose a uniform sub-band quantization of the Fresnelet coefficients followed by the lossless Burrows-Wheeler transform. Another method proposed in the same work is the Set Partitioning in Hierarchal trees (SPIHT) coding algorithm on the Fresnelet coefficients.

\section{GABOR WAVELETS}

Gabor wavelets are finding increasing use in the field of many computer vision applications and modelling of biological vision. Gabor wavelets provide for scaling and rotation along with translation, and hence a very well suited for measuring the local spatial frequencies of holograms. The uncertainty bound plays an important role in time-frequency localization. It is known that the Gaussian function ideally provides the best uncertainty bound. Gabor functions are in essence Gaussian functions which are modulated with a sinusoidal function.

The main objective of a wavelet transform, is to obtain the best approximation of the hologram in the hologram plane. Gabor showed that the minimal area which reflects the trade off between time resolution and frequency resolution, has a lower bound in their product, which is analogues to the uncertainty product in physics. He discovered that the Gaussian modulated complex exponentials provide the best trade-off.

Let $g(x, y)$ represent the mother wavelet, then a set of Gabor functions $g_{s, \theta}(x, y)$ can be generated by rotating (using parameter $\theta$ ) and scaling (using parameter $s$ ) $g(x, y)$ to form an almost complete non-orthonormal basis set

$$
g_{s, \theta}(x, y)=c^{-2 s} g\left(x^{\prime}, y^{\prime}\right)
$$

where $x^{\prime}=c^{-s}(x \cos \theta+y \sin \theta), y^{\prime}=c^{-s}(-x \sin \theta+y \cos \theta)$ and $c$ is selected based on the frequency centering of the Gaussian function and the scaling parameter $s$.

The basic Gabor function is represented by using 2 operators:

1) The modulation operator $E_{b} \mid\left(E_{b} g\right)(x):=e^{i 2 \pi b x}, b>0$

2) The translation operator $T_{a} \mid\left(T_{a} g\right)(x):=g(x-a), a>0$

$$
\left\{E_{b} T_{a} g\right\}=e^{i 2 \pi b x} g(x-a)
$$

Hence the overall gabor wavelet is composed of 4 tweak-able parameters for modulation, translation, scaling and rotation and the wavelet is denoted as $g_{s, \theta, a, b}(x)$. Usually the selection of $a, b$ form an important role in the selection of the wavelet. The frame bounds $A, B$ are dependant on these values. The famous result of Gabor frames states that $\left\{E_{b} T_{a} g\right\}$ can be a frame for $L_{2} \mathbb{R}$ only if $a b \leq 1$. If $a b=1$ then it forms a Riesz basis. Hence we need select $a$ and $b$ such that $a b<1$, so that the above mentioned Balian-Low theorem does not affect us.

It is also known that the Gabor wavelet transform is not reversible. In other words we know that at 0 frequency we will get an non-zero output. The parameters of the Gabor wavelet can be selected in such a way, that this limitation can be overcome. If the modulation frequency is selected to be large, then the output at 0 frequency tends to 0 . Hence we select the modulation parameter $b=1$, which is the frequency $2 \pi$.

We consider the wavelet equation provided in ${ }^{6}$ for our reconstruction process.

$$
\psi(x)=\frac{1}{\sqrt[4]{\pi}} \sqrt{\frac{2 \pi}{\gamma}} \exp \left[-\frac{(2 \pi / \gamma)^{2} x^{2}}{2}+j 2 \pi x\right]
$$

Here it is observed that $a \approx 0.55$ and $b=1$ when $\gamma=\pi \sqrt{2 / \ln 2}$. The other features of this wavelet equation are that it is centered at $x=0$ at frequency $\omega=2 \pi$. Then the function $\psi_{s, a}(x-k)$, which is the scaled and translated version of this wavelet will be centered at $x=k$ and $\omega=\frac{2 \pi}{s}$, with a full width of half maximum of $\Delta x=2 s$. 

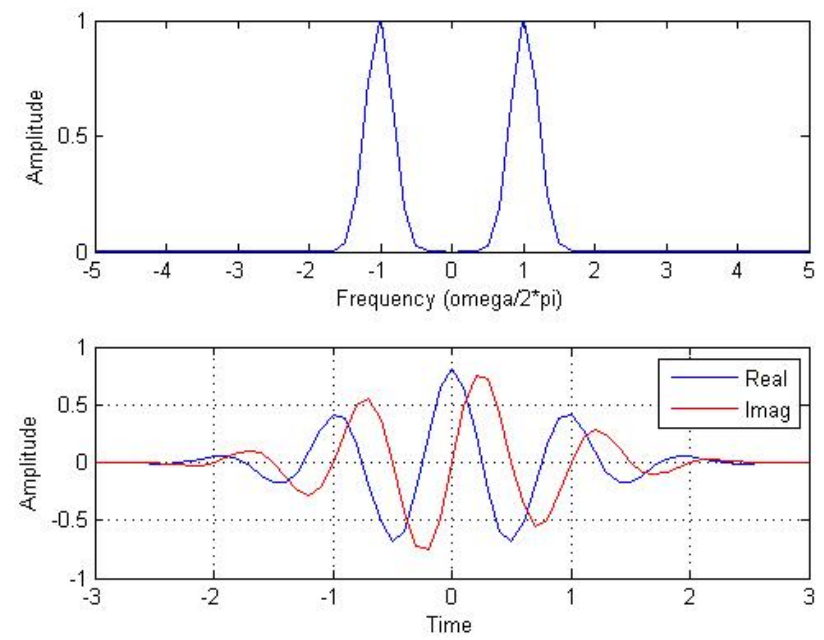

Figure 3. Gabor wavelet basis showing the Frequency (top) and Time domain representations

There is another way by which one can circumvent the Balian-Low theorem. Daubechies et.al ${ }^{15}$ proved that if one is ready to give up the Gabor structure, it is possible to obtain a well localized orthonormal basis: if $g \in L_{2} \mathbb{R}$ is chosen such that $\hat{g}$ is real valued and $\left\{E_{b} T_{a / 2} g\right\}$ is a frame with bounds $A=B=2$, then the collection of functions $\left\{\psi_{l, k}\right\}_{l \geq 0, k \in \mathbb{Z}}$ constitutes an orthonormal basis for $L_{2} \mathbb{R}$. These bases are called Wilson bases. The trick is to alternately use Sine and Cosine functions for odd and even coefficients instead of the complex exponentials. This is sufficient to beat the Balian-Low condition.

But since we intend to use the 2D Gabor wavelet transform, which has been shown to provide better results by, ${ }^{6}$ the use of complex exponentials in the calculation of coefficients becomes necessary. Mathematically, the 2D Gabor function achieves the time-frequency resolution limit only in its complex form, since a complex valued 2D Gabor function contains in quadrature projection an even-symmetric cosine component and an odd-symmetric sine component. ${ }^{16}$

Figure 3 shows a well localized Gabor wavelet centered at the frequency $2 \pi$.

Once we have the Gabor wavelet transformed coefficients, these can be used for view-based pruning techniques like the one explained in Section 3. Also it observed that the Gabor wavelet bases are well localized in the spatial domain as compared to Fresnelets. Hence the approximation of a local area in the hologram using these wavelet bases will result in better extraction of features for reconstruction based on view-dependent compression.

One good feature of 2D Gabor wavelets is that it provides natural suppression of the unwanted orders. The Gabor wavelet used for our experiment is the one given in. ${ }^{6}$

$$
\psi\left(\frac{x-a}{s}, \frac{x-b}{s}, \theta\right)=\frac{1}{\sqrt[4]{\pi}} \sqrt{\frac{2 \pi}{\gamma}} \exp \left\{-\frac{(2 \pi / \gamma)^{2}\left[(x-a)^{2}+(y-b)^{2}\right]}{2 s^{2}}\right\} \cdot \exp \left\{j 2 \pi \frac{(x-a) \operatorname{Cos} \theta+(y-b) \operatorname{Sin} \theta}{s}\right\}
$$

$s$ represents the scaling of the wavelet function and $\theta$ represents the rotation. $\gamma$ is selected to be $\pi \sqrt{2 / \ln 2}$. We obtain the Gabor wavelet transform for every $s$ and $\theta$ value based on the translation parameters $a$ and $b$ for a hologram.

The peak of the 2D Gabor wavelet transform is obtained where the magnitude of the wavelet coefficient is maximum for every $s$ and $\theta$, at every $a$ and $b$ value in the hologram. This is called as the ridge of the Gabor wavelet transform. It has been shown that the wavelet coefficient constructed in this manner is equal to the object wave at the hologram plane multiplied by a constant. This method also has shown that there is natural suppression of the zero-order and real images with out the need of any spatial filtering.

Figure 4 gives the $\psi$ functions for $5 s$ values at intervals of 1 and 10 rotation values at intervals of 18 degrees. 


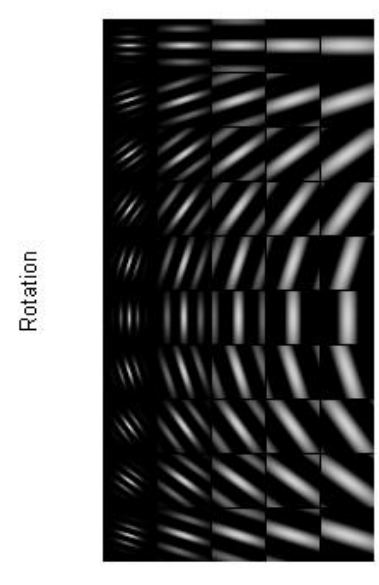

Scale

Figure 4. 2D Gabor wavelets for 5 different scales and 10 different rotations

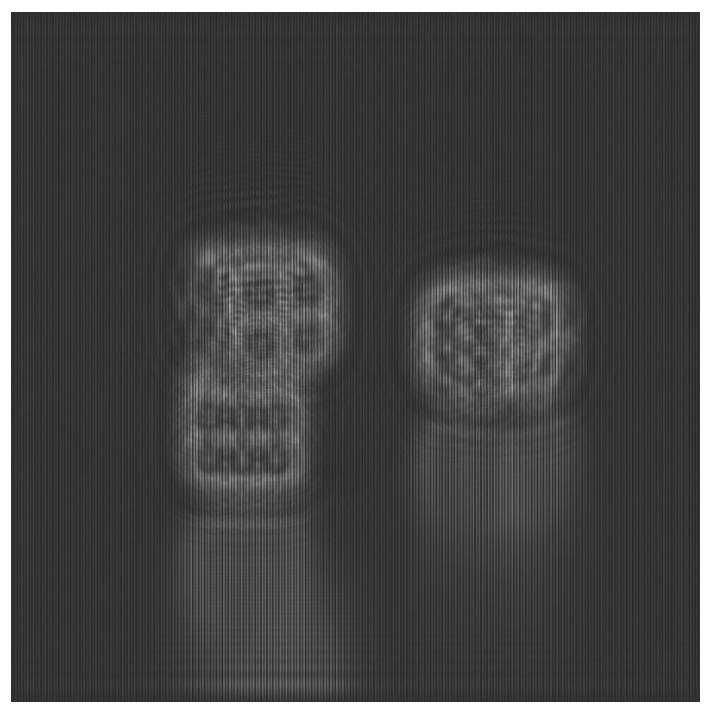

Figure 5. Hologram of an image of some dice. (Original Image courtesy Remi Rouaud, France Telecom OLPS)

\section{RESULTS}

Using the two wavelets we obtained the reconstruction of a hologram. For Gabor wavelets we used the Angular Spectrum method to perform the reconstruction. For Fresnelets as usual it is the Fresnel transform. The hologram was generated numerically in off-axis configuration with a separation of 2 degrees between reference wave and object wave Figure 5 .

Gabor wavelets show a suppression of the unwanted orders if the wavelet coefficients are calculated at the ridge of the transform. cf. Figure 7. The output of a Fresnelet transformed image in Figure 6 shows unwanted orders being generated.

The code for both the transforms is written in Matlab@), and the results are generated on a PC with a Xeon (C)E-1620 CPU running at 3.6 Ghz with 8 GB of RAM. The hologram in Figure 5 is of 1024x1024 size. The Fresnelets code in its unoptimised form performs the transform in 25 seconds while the unoptimised 2D Gabor Wavelet transform code, takes about 4 minutes for completion. This is evident from the fact that each 


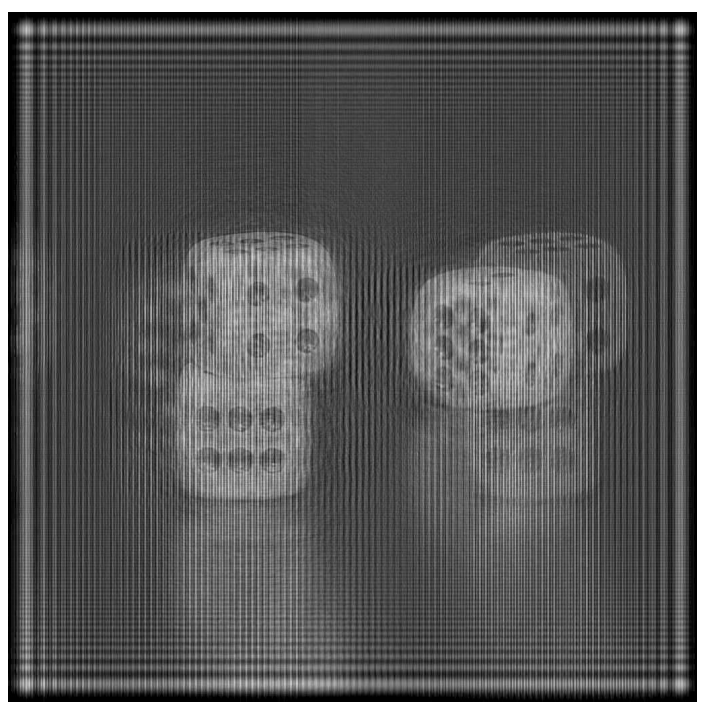

Figure 6. Fresnelet transformed reconstruction of the hologram. Note the unwanted order are visible in the reconstruction. Output is centerd on the DC term with the real image and virtual image on the left and right sides respectively.

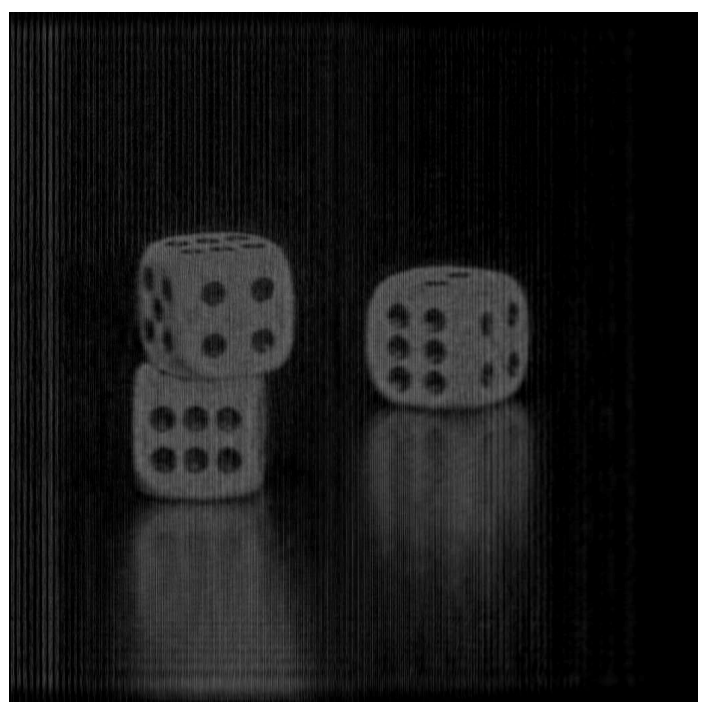

Figure 7. Reconstruction of a Gabor transformed hologram using Angular Spectrum method. Notice that the unwanted orders are suppressed. Output is centered on the virtual image. 
rotation angle and scale of the gabor wavelet produces one transformed image. Later the maximum of the gabor coefficients is found, to obtain the values at the ridge of the transform.

\section{CONCLUSION}

In this paper view-based compression techniques have been discussed and the importance of having good localization for the wavelet has been emphasized. Two wavelet bases (Fresnelets and Gabor wavelets) have been compared on the basis of space - frequency localization. It has been experimentally shown that Gabor wavelets are able to suppress the unwanted orders created in the reconstruction process without the need for spatial filtering. Gabor wavelets are more computationally complex than Fresnelets, hence optimisation of the Gabor wavelet transform using multi-core or multi processor architectures becomes necessary. The good localization properties of the gabor wavelet bases in space and frequency domain, make them an easy choice for view-based compression techniques for digital hologram reconstruction. However, it is at the cost of heavy computional complexity at encoding stage, and this has to be studied further to possibly exploit the parallel character of analysis and provide efficient GPU implementations.

\section{REFERENCES}

[1] Darakis, E. and Naughton, T. J., "Compression of digital holograms sequences using mpeg-4," Proc. of SPIE 7358 (March 2009).

[2] Shortt, A., Naughton, T., and Javidi, B., "Compression of digital holograms of 3d objects using wavelets," Optics Express 2625 14 (April 2006).

[3] Halle, M. W., The Generalized Holographic Stereogram, Master's thesis, Massachusetts Institute of Technology (1991).

[4] Smithwick, Q., Barabas, J., Smalley, D., and Jr, M. B., "Interactive holographic stereograms with accommodation cues," Object based media group, MIT Labs 15 (December 2009).

[5] Liebling, M., Blu, T., and Unser, M., "Fresnelets: New multiresolution waveletbases for digital holography," IEEE Transactions on Image Processing 12, 29-43 (January 2003).

[6] Zhong, J. and Weng, J., [Holography, Research and Technologies, Prof. Joseph Rosen (Ed.)], Intech Open (2011).

[7] Schwerdtner, A., Hussler, R., and Leister, N., "Large holographic displays for real-time applications," Journal of Display Technology 6912 (January 2008).

[8] Y, F., Kang, H., and Onural, L., "State of the art in holographic displays: A survey," Journal of Display Technology 6, 443-454 (October 2010).

[9] Onural, L., "Diffraction from a wavelet point of view," Optics Letters 18 (June 1993).

[10] Li, Y., Szu, H., Sheng, Y., and Caulfield, J., "Wavelet processing and optics," IEEE Invited paper 84 (May 1996).

[11] Onural, L. and Kocatepe, M., "Family of scaling chirp functions, diffraction and holography," IEEE Transactions on Signal Processing 43 (July 1995).

[12] Unser, M., Aldroubi, A., and Eden, M., "On the asymptotic convergence of b-splines wavelets to gabor functions," IEEE Transactions on Information theory 38, 554-573 (March 1992).

[13] J., B. J., Christopher, H., and F, W. D., "Differentiation and the balian?low theorem," Journal of Fourier Analysis and Applications 1 (December 1994).

[14] E, D. and JJ, S., "Use of fresnelets for phase-shifting digital compression," IEEE Transactions in Image Processing 15 (December 2006).

[15] Daubechies, I., Jaffard, S., and Journes, J.-L., "A simple wilson orthonormal basis with exponential decay," SIAM J MATH 22, 554-573 (March 1991).

[16] Lee, T. S., "Image representation using 2d gabor wavelets," IEEE transaction on Pattern Analysis and Machine Learning 18, 1-13 (october 1996). 